\title{
The Efficacy of Manual Axially Loaded Magnetic Resonance Imaging in Diagnosing Potential Thoracolumbar Osteoporotic Fracture Instability: A Case Report
}

\author{
Tarik Yazar ${ }^{1}$, Lawrence A. Delasotta ${ }^{*}, 2$, Adam Pearson ${ }^{3}$, Gursukhman Sidhu ${ }^{4}$, Jordan Gruskay ${ }^{4}$, \\ Alexander Vaccaro ${ }^{4}$, Warren Pettine ${ }^{5}$, Kris Radcliff ${ }^{4}$, Chris Kepler ${ }^{4}$ and Kenneth Pettine ${ }^{5}$ \\ ${ }^{1}$ University of Ankara School of Medicine, Traumatology, Ankara, Turkey \\ ${ }^{2}$ Temple University, Department of Surgery, Philadelphia, Pennsylvania, USA \\ ${ }^{3}$ Dartmouth-Hitchcock Medical Center, Orthopaedic Surgery, Lebanon, New Hampshire, USA \\ ${ }^{4}$ Thomas Jefferson University, Orthopaedic Surgery, Philadelphia, Pennsylvania, USA \\ ${ }^{5}$ The Spine Institute, Orthopaedic Surgery, Loveland, Colorado, USA
}

\begin{abstract}
A 68 year old female who had occult instability at a symptomatic osteoporotic L1 burst fracture was diagnosed by manual axially loaded magnetic resonance imaging. Traction resulted in the reduction of the fracture and decompression of the conus. Based on these findings, the patient underwent L1-L2 fusion with complete resolution of her back and leg pain. Our objective is to describe the utility of such imaging in detecting an occult lumbar instability. This study suggests that a manual axially loaded compression-traction magnetic resonance image may be an efficacious diagnosis option in patients with persistent symptoms treated non-operatively.
\end{abstract}

Keywords: Dynamic magnetic resonance imaging, compression fracture, thoracolumbar fracture, vertebroplasty, percutaneous cement stabilization.

\section{INTRODUCTION}

The use of axial loading during MRI is a topic of interest in diagnostic spine imaging. The technique may capture subtle anatomic changes on MRI in both degenerative and traumatic disorders. In theory, an axially applied compressive load should reproduce the patient's symptoms if it results in compression of neural elements. The manual

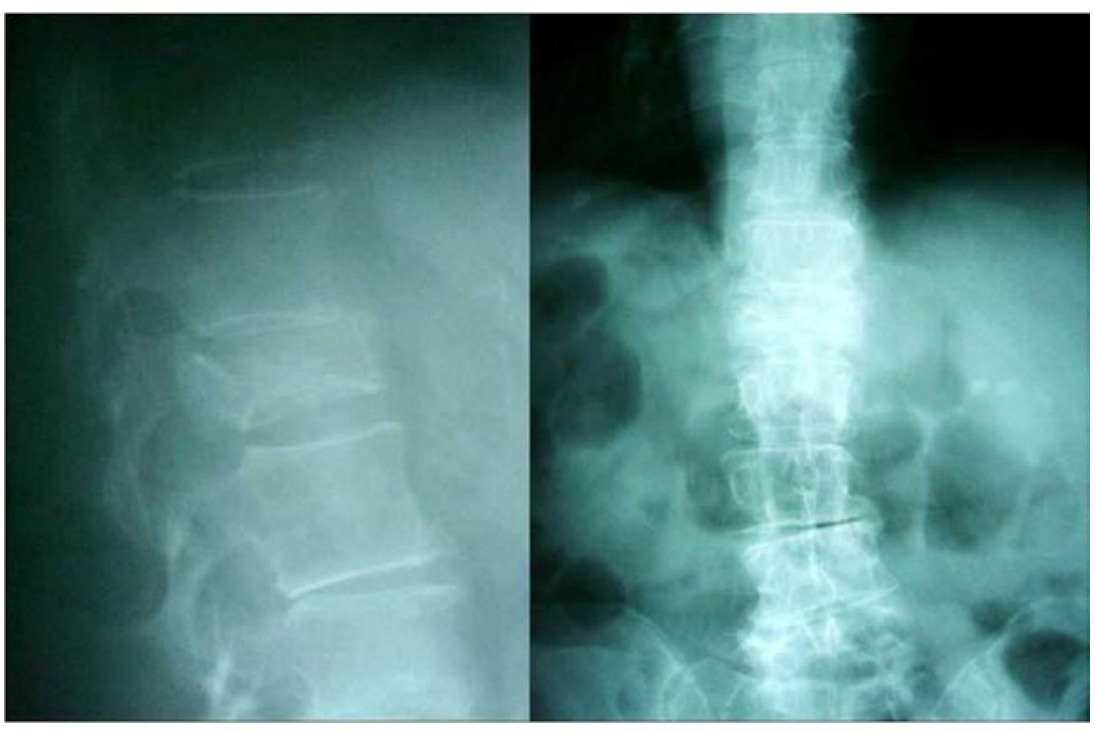

Fig. (1). AP and lateral plain film of unstable osteoporotic L1 burst fracture prior to vertebroplasty.

*Address correspondence to this author at the $3401 \mathrm{~N}$ Broad Street, Parkinson Pavilion, Suite 400, Philadelphia, PA, 19140, USA; Tel: 215707-5080; Fax: 215-707-1915; E-mail: LawrenceDelasotta@gmail.com technique described in this case study - performed due to lack of a formal axial loading device - may be an efficacious alternative to more expensive equipment to demonstrate dynamic instability. The majority of published literature on 


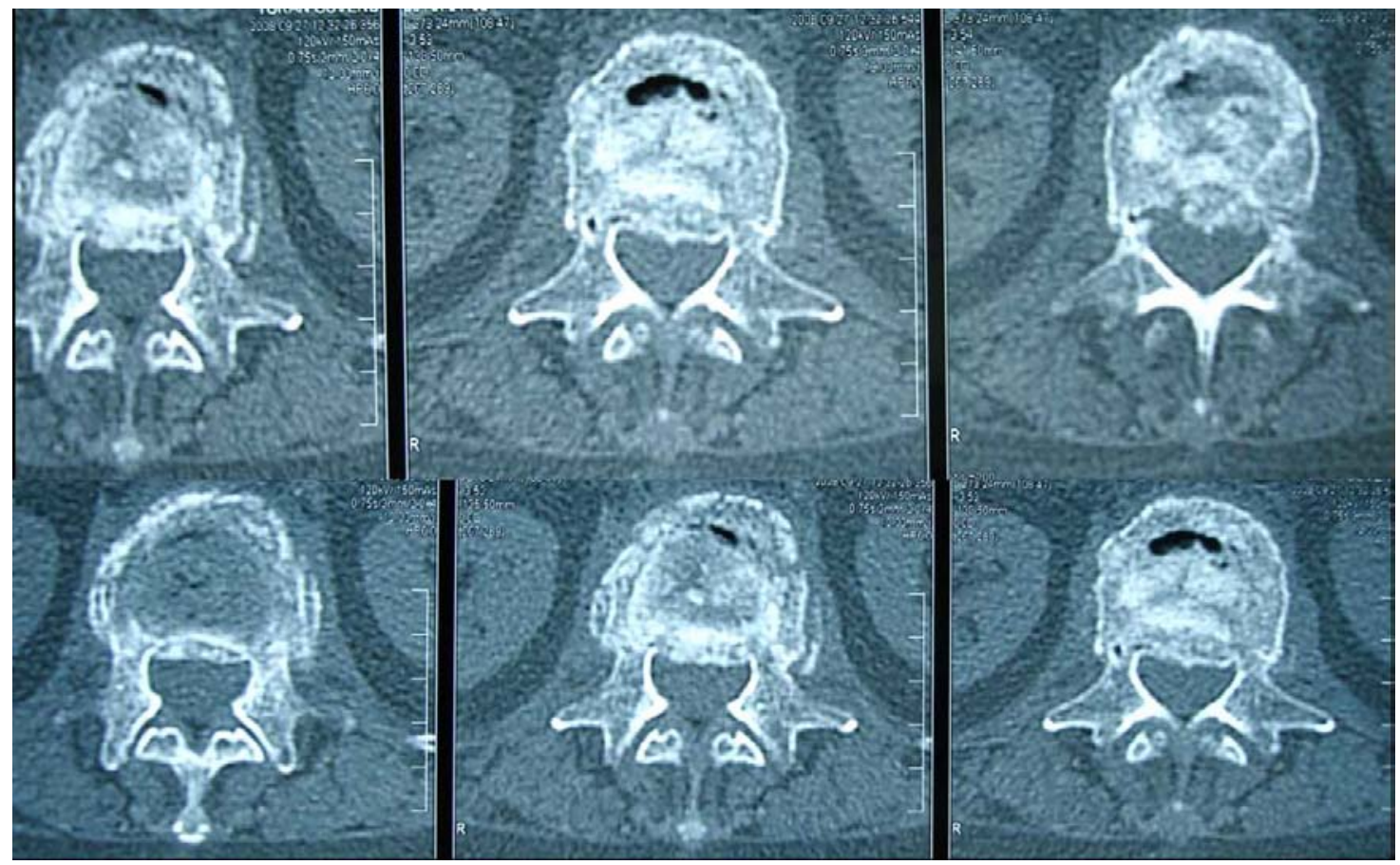

Fig. (2). Axial CT scans demonstrate through L1-2 no significant canal compromise, fractured cement, and extensive callus.

this topic reported results using a DynaWell device (DynaWell L-spine, DynaWell Inc. Las Vegas, USA), which applied a compression-traction load that is approximately $50 \%$ of an individual's body weight $[1,2]$.

The purpose of this case report is to describe the use of manual axial loading during MRI to evaluate abnormal motion of a vertebral body in a patient with occult thoracolumbar instability. It also may demonstrate that manual axial loading is as efficacious as more costly dynamic imaging technologies.

\section{CASE REPORT}

The authors have obtained the patient's informed written consent for print and electronic publication of this case report.

A 68 year old female was referred to our practice three months after a percutaneous cement stabilization for an L1 burst fracture - she provided no images of soft tissue or posterior ligamentous complex integrity prior to stabilization (Fig. 1). The patient reported symptoms of severe back pain, shooting pains down both legs when upright, tenderness at the fracture level, balance and urinary problems - all of which remained the same or worsened after percutaneous injection. Pain when standing discouraged the use of upright flexion-extension films to characterize the injury; however, axial CT scans were obtained (Fig. 2).

For standard recumbent MRI, a Magnetom Avanto, 1.5 Tesla Tim MRI system (Siemens Healthcare, Erlangen, Germany) was used. The settings were: T2W sagittal TR:3210 ms, TE:107 ms; T2W axial TR:3210 ms, TE:122 ms; and T1W sagittal TR:538 ms, TE:10 ms. The standard recumbent MRI failed to demonstrate any evidence of spinal cord or nerve root compression, nor did it reveal a fracture following cement stabilization (Fig. 3).
A manually applied axial compression-traction force was applied in a carefully monitored setting by two people (one applying pressure at the shoulders and the other at the ankles) for six minutes while $\mathrm{T} 2$ images were obtained - a technician pushed/pulled for six minutes at approximately $20 \mathrm{~kg}$ each for a combined total of $40 \mathrm{~kg}(\sim 1 / 2$ body weight). The same MRI scanner was used as described above, with the following settings to obtain axially loaded images: T2W sagittal TR:3490 ms, TE:107 ms.

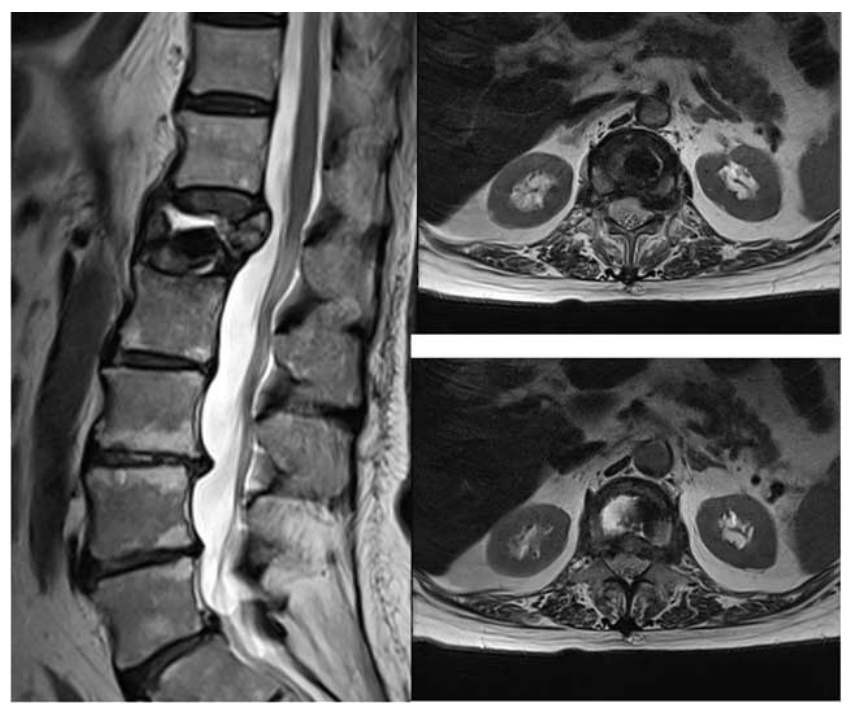

Fig. (3). Sagittal and Axial T2 weighted MRI demonstrating unstable compression fracture with cement fixation.

The traction images demonstrated reduction of the fracture fragment as well as decompression of the conus, with an increase in vertebral body height due to ligamentotaxis (Fig. 4a,b). Thus, the axial loading dynamic images identified gross spinal instability not appreciated on the standard recumbent images. An L1-L2 posterolateral instrumented 


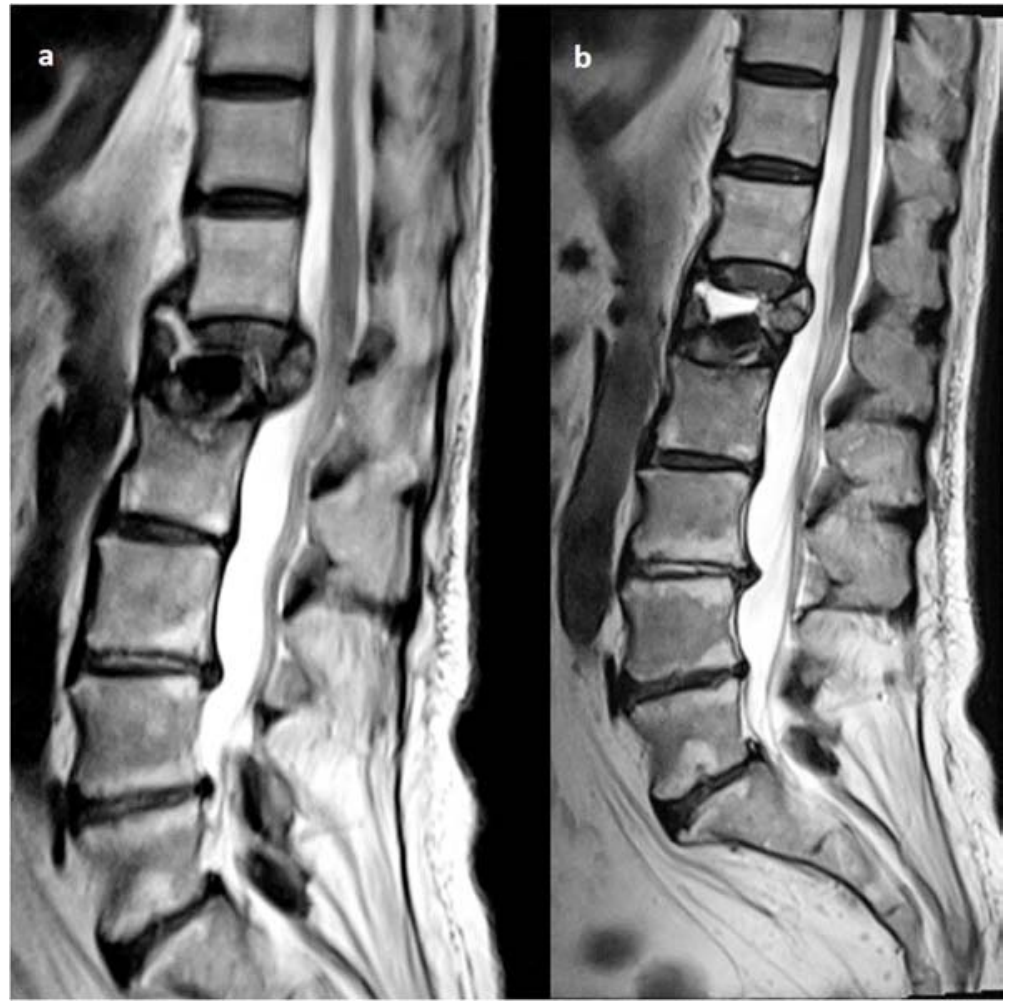

Fig. (4). Sagittal T2 weighted MRI's demonstrating dynamic axial compression (a) and axial traction (b) images with prior vertebroplasty at L1.

fusion was then performed to stabilize the fracture. A distraction force was applied across the pedicle screws in order to obtain the reduction noted on the traction MRI.

The Turkish Oswestry Disability Index (TODI) [3, 4] revealed a range of $60-80 \%$ preoperatively - demonstrating severe disability. Six weeks postoperatively, the TODI was $0-20 \%$, and at eighteen weeks $0 \%$. By 12 weeks the patient was pain free and back to regular activities without limitation. This case elegantly demonstrates the ability of manual dynamic MRI to detect a subtle fracture instability not demonstrated on static MRI. In this case, the treatment plan was modified based on the result of the dynamic MRI with a marked improvement in the patient's symptoms.

\section{DISCUSSION}

This case study suggests that a manual axially loaded compression-traction MRI may be an efficacious option to identify an occult spinal instability or stenosis that may not be demonstrated on a standard recumbent MRI. This imaging modality may be indicated in a patient with persistent symptoms following appropriate non-operative treatment of a known osteoporotic thoracolumbar burst fracture. Furthermore, the use of manual compressiontraction may present an opportunity to reduce health care cost.

Saifuddin et al. conducted a literature review of axial loaded MRIs, which focused on cadaveric studies that used the DynaWell device [5]. They concluded that axial loading resulted in central canal, lateral recess and foraminal dimension reductions but did not consistently result in neural compression or nerve root displacement. However, axial loading did result in a statistically significant decrease in dural sac cross-sectional area. In regards to disk herniation, an axially loaded MRI demonstrated an increase in the size of the herniation in $20-50 \%$ of patients [6].

While studying the mechanical responses of the lumbar spine to axial compression in asymptomatic individuals, Wisleder et al. found that, on average, compression did not result in a significant decrease in the measured length of the spine [7]. Several individuals, however, showed a marked decrease or increase in spine length under compression, with the quantity and direction of change varying substantially. Those researchers used $100 \%$ body weight applied for 6.5 minutes. Interestingly, the spinal alignment changes they noted were most common at the thoracolumbar junction (T12-L1) - which is not only the location of injury in our case study but also the level at which burst fractures most commonly occur [7].

Danielson et al. applied a compressive force of 0.25 to 0.5 times body weight to the lumbar spines of individuals with symptomatic and asymptomatic spinal stenosis using the DynaWell device [2, 8, 9]. The authors found that an axially loaded MRI demonstrated increased stenosis in $69 \%$ of patients with neurogenic claudication and in $14 \%$ of patients with symptomatic sciatica compared to static MRI, but demonstrated no additional pathology in patients with symptomatic low back pain. In 2008, Willén et al. [8] evaluated the surgical outcomes from procedures relying on axially loaded MRI for the diagnosis of stenosis with a follow up of 1-6 years. At follow-up, $76 \%$ of patients had little remaining leg pain (VAS less than 25 out of 100). Leg 
pain improved in $96 \%$ of patients, and all but one patient were able to walk.

Hansson et al. studied lumbar spinal canal narrowing during axial compression in 24 patients. They found that axial loading of the spine with DynaWell typically resulted in ligamentum flavum infolding while less frequently causing increased disc bulging [10]. Jinkins et al. conducted a study using standing dynamic MRI to evaluate the cervical and lumbar spine. They found that this imaging modality detected disk herniations, spinal stenosis, and intersegmental listhesis more frequently than conventional recumbent MRI [11]. In contrast, Madsen et al. measured lumbar lordosis and disk height using the DynaWell loading machine at 40$50 \%$ of bodyweight and found that the standing and axially loaded MRI were no more effective in diagnosing lumbar spinal stenosis than the standard recumbent MRI [12].

The present literature has a few studies that demonstrate the utility of dynamic MRI in the detection of spinal stenosis and spondylolisthesis not present on a static, recumbent MRI. To our knowledge, this is the first case report of the efficacy of a manual axially loaded dynamic MRI demonstrating instability. Furthermore, this report may demonstrate that manual traction may be as efficacious as an expensive compression-traction imaging device. Further study may be warranted to detect the sensitivity of manual axial loaded imaging relative to the expensive compression-traction devices.

In conclusion, this case study suggests that dynamic MRI may be useful to detect subtle instability in patients with persistent symptoms following osteoporotic thoracolumbar fractures. In this case, an axial loaded MRI identified occult instability in a patient with percutaneous cement stabilization of an osteoporotic L1 burst fracture suffering from lumbar radiculopathy and back pain. Subsequent surgical treatment resulted in a complete recovery. A future prospective study evaluating the usefulness of manual dynamic MRI in patients with persistent symptoms despite appropriate non-operative treatment for thoracolumbar fractures may be warranted. Furthermore, a cost-benefit analysis may show an opportunity to reduce health care cost.

\section{REFERENCES}

[1] Madsen R, Jensen T, Pope M, Sørensen J, Bendix T. The effect of body position and axial load on spinal canal morphology: an MRI study of central spinal stenosis. Spine 2008; 33(1): 61-7.

[2] Willén J, Wessberg P, Danielsson B. Surgical results in hidden lumbar spinal stenosis detected by axial loaded computed tomography and magnetic resonance imaging: an outcome study. Spine 2008; 33(4): E109-15.

[3] Fairbank J, Couper J, Davies J, O'brien J. The Oswestry low back pain disability questionnaire. Physiotherapy 1980; 66(8): 271-3.

[4] Fritz J, Irrgang J. A comparison of a modified Oswestry low back pain disability questionnaire and the Quebec back pain disability scale. Phys Ther 2001; 81(2): 776-88.

[5] Saifuddin A, Blease S, MacSweeney E. Axial loaded MRI of the lumbar spine. Clin Radiol 2003; 58(9): 661-71.

[6] Saifuddin A, McSweeney E, Lehovsky J. Development of lumbar high intensity zone on axial loaded magnetic resonance imaging. Spine 2003; 28(21): E449-51.

[7] Wisleder D, Smith M, Mosher T, Zatsiorsky V. Lumbar spine mechanical response to axial compression load in vivo. Spine 2001; 26(18): E403-9.

[8] Willén J, Danielson B. The diagnostic effect from axial loading of the lumbar spine during computed tomography and magnetic resonance imaging in patients with degenerative disorders. Spine 2001; 26(23): 2607-14.

[9] Willén J, Danielson B, Gaulitz A, Niklason T, Schönström N Hansson T. Dynamic effects on the lumbar spinal canal: axially loaded CT-myelography and MRI in patients with sciatica and/or neurogenic claudication. Spine 1997; 22(24): 2968-76.

[10] Hansson T, Suzuki N, Hebelka H, Gaulitz A. The narrowing of the lumbar spinal canal during loaded MRI: the effects of the disc and ligamentum flavum. Eur Spine J 2009; 18(5): 679-86.

[11] Jinkins J, Dworkin J, Damadian R. Upright, weight-bearing, dynamic-kinetic MRI of the spine: initial results. Eur Radiol 2005; 15(9): 1815-25.

[12] Madsen R, Jensen T, Pope M, Sørensen J, Bendix T. The effect of body position and axial load on spinal canal morphology: an MRI study of central spinal stenosis. Spine 2008; 33(1): 61-7. 